Research Article

\title{
Structural Response Analysis under Dependent Variables Based on Probability Boxes
}

\author{
Z. Xiao and G. Yang \\ State Key Laboratory of Advanced Design and Manufacturing for Vehicle Body, College of Mechanical and Vehicle Engineering, \\ Hunan University, Changsha 410082, China
}

Correspondence should be addressed to G. Yang; yanggang@hnu.edu.cn

Received 29 October 2014; Revised 16 March 2015; Accepted 7 April 2015

Academic Editor: Alessandro Palmeri

Copyright (c) 2015 Z. Xiao and G. Yang. This is an open access article distributed under the Creative Commons Attribution License, which permits unrestricted use, distribution, and reproduction in any medium, provided the original work is properly cited.

\begin{abstract}
This paper considers structural response analysis when structural uncertainty parameters distribution cannot be specified precisely due to lack of information and there are complex dependencies in the variables. Uncertainties in parameter are quantified by probability boxes ( $p$-boxes) and dependence among uncertain parameters is modeled by copula. To calculate uncertainty structural response, a sampling-based method is proposed. In this method, a sampling strategy is used to sample random intervals from dependent $p$-boxes according to the copula theory and the metamodel-based optimization method is applied to solve a range of structural interval response problems. Two types of errors are presented to evaluate the error of different $p$-boxes. Four numerical examples are investigated to demonstrate the effectiveness of the present method.
\end{abstract}

\section{Introduction}

Traditional quantification of the uncertainties existing in a system and the corresponding calculation of uncertainty propagation are generally based on the probability model, in which random distributions are used to describe the uncertainty. Unfortunately, for practical engineering problems, sufficient experimental samples are not always available or sometimes very expensive to obtain. Thus alternative imprecise probabilities have been proposed, including the interval theory [1], the evidence theory [2], and probability boxes ( $p$ boxes) [3]. The focus of this paper is on $p$-boxes, which are more expressive generalization of both traditional probability distributions and interval representations. The $p$-box incorporates facilities from probability theory for modeling correlations and dependencies and from interval analysis expressing ignorance by expressing interval bounds on the cumulative distribution function (CDF) for a random variable [4]. On the other hand, the $p$-box has a clear behavioral interpretation and therefore is easy acceptable for practicing engineers [5].

In the field of uncertainty analysis based on $p$-boxes, a lot of exploratory work has been reported in theoretical and application aspects. For example, Williamson and Downs [6] proposed an algorithm to compute binary arithmetic operations (addition, subtraction, multiplication, and division) on pairs of independent discrete $p$-boxes for risk assessment. Karanki et al. [7] used the two-phase Monte Carlo simulation to calculate the multiplication of two $p$ boxes for the safety assessment. Bruns [8] compared three uncertainty propagation methods to find the appropriate computational methods for propagating $p$-boxes through the black box engineering models. Zhang et al. [9-11] combined the interval finite element analysis (FEA) and the interval sampling method and proposed an interval Monte Carlo (IMC) method for structural analysis with $p$-boxes which are defined by distributions with interval parameters. Bai et al. [12] developed a numerical method to compute the linear elastic static and dynamic response of structures with epistemic uncertainty represented by evidence variables, which integrated the moment concept and finite element method and also suited to $p$-boxes variables. Ghosh and Olewnik [13] improved the performance of the two-phase Monte Carlo simulation by replacing the outer loop by optimization algorithms and replacing the inner loop by the sparse grid numerical integration. 
Among the above-mentioned methods, they focused on discussing uncertainty propagation of independent $p$ boxes. However, the complex dependencies are common in physical systems and can have profound impacts on the numerical results of calculations [14-16]. Ferson et al. [17] illustrated several methods which are used to model the dependence among variables that are cannot be generalized easily for use with $p$-boxes and proposed that copulas could represent easily the dependence in $p$-boxes. Based on the analysis, they presented an approach known as the dependency bounds convolution (DBC) method. The DBC method could calculate the propagation of dependent $p$-boxes whose dependence is expressed by given parametric copula. Despite the fact that DBC is useful for a sequence of basic arithmetic operations or elementary function with a small number of uncertain variables, it must overcome some obstacles for application in engineering design. Among these problems, the low efficiency difficulty seems to be the severest one. The Cartesian product method is applied in the DBC, which can impose significant computational burden.

In this paper, a computational method is proposed to propagate dependent $p$-boxes through structural model, where dependence in $p$-boxes is described by copula. By the copula sampling technique, the input epistemic uncertainties represented by $p$-boxes are transformed into a range of intervals. The metamodel-based optimization is employed as the solver to calculate the structural response with input intervals. A mathematical function has been tested to show the procedure and the efficiency of the proposed method and then three structural numerical examples are investigated by the present method.

\section{Probability Boxes Theory under Dependence}

2.1. Probability Boxes. A $p$-box is a class of distribution functions delimited by an upper and a lower bound which collectively represent the epistemic uncertainty about the distribution function of a random variable. Let $\mathbf{D}^{P}$ denote the space of distribution functions on the real numbers $\mathfrak{R}$. A $p$-box will be defined as

$$
\mathbf{D}^{P}=\{F(x): \forall x \in \Re, \underline{F}(x) \leq F(x) \leq \bar{F}(x)\},
$$

where $\underline{F}(x), F(x), \bar{F}(x): \Re \rightarrow[0,1], \underline{F}=P(X \leq x)$, and $\bar{F}=$ $P(X \leq x)$ are the lower and upper bounds of a $p$-box, which is also written as $[\bar{F}, \underline{F}]$.

There are several ways to construct $p$-boxes, depending on the type of information available. In this paper, $p$-boxes are constructed based on $95 \%$ confidence intervals on the parameters of a known distribution type. The distribution types are determined on the basis of the theoretical knowledge or previous experience.

2.2. Describing Dependence in Probability Boxes with Copula. Now, consider the dependence in $p$-boxes which can be described by copulas. A copula is a multivariate probability distribution for which the marginal probability of each variable is uniformly distributed. By Sklar's theorem [18], copulas are simply the dependence functions that knit together marginal distributions to form their joint distribution. Consider a random vector $\left(X_{1}, X_{2}, \ldots, X_{d}\right)$ with CDFs $\left(F_{1}\left(x_{1}\right)\right.$, $\left.F_{2}\left(x_{2}\right), \ldots, F_{d}\left(x_{d}\right)\right)$ which are represented by $p$-boxes in this paper, the multivariate cumulative distribution function can be written as

$$
H\left(x_{1}, x_{2}, \ldots, x_{d}\right)=C\left(F_{1}\left(x_{1}\right), F_{1}\left(x_{2}\right), \ldots, F_{d}\left(x_{d}\right)\right),
$$

where $C$ is a multivariate copula.

2.3. Dependency Bounds Convolution Method. Ferson et al. [17] firstly used dependency bounds convolution method to calculate binary arithmetic operations on pairs of dependent discrete Dempster-Shafter structures. This method mainly contains discretization of the $p$-boxes and Cartesian product. Consider that $f: \mathbf{X} \rightarrow Y, \mathbf{X}=\left(X_{1}, X_{2}\right)$ are two dependent $p$-boxes connected by copula function $C\left(X_{1}, X_{2}\right)$, and $Y$ is the response quantity. The $p$-boxes $X_{1}$ and $X_{2}$ are discretized into $M$ focal elements, which are represented by $\left\{\left(\left[a_{i}^{L}, a_{i}^{R}\right], p_{i}\right)\right\}(i=1,2, \ldots, M)$ and $\left\{\left(\left[b_{j}^{L}, b_{j}^{R}\right], q_{j}\right)\right\}(j=$ $1,2, \ldots, M)$, respectively. By Yager's Cartesian product, the response quantity in the $(i, j)$ th focal element can be defined by an interval $\left[z_{l}^{L}, z_{l}^{R}\right]$, where $l=M *(i-1)+j$. The probability mass $r_{l}$ associated with the $(i, j)$ th focal element can be calculated by

$$
\begin{aligned}
r_{l}= & C\left(P_{i}, Q_{j}\right)-C\left(P_{i-1}, Q_{j}\right)-C\left(P_{i}, Q_{j-1}\right) \\
& +C\left(P_{i-1}, Q_{j-1}\right),
\end{aligned}
$$

where $P_{i}$ and $Q_{j}$ are the cumulative masses, $P_{i}=\sum_{k=1}^{i} p_{k}$ and $Q_{i}=\sum_{k=1}^{j} q_{k}$. The result is written as focal elements $\left\{\left(\left[z_{l}^{L}, z_{l}^{R}\right]\right.\right.$, $\left.\left.r_{l}\right)\right\}\left(l=1,2, \ldots, M^{2}\right)$ which can be transferred into a $p$-box by

$$
\begin{aligned}
& \bar{F}(z)=\sum_{z_{l}^{L} \leq z} r_{l}, \\
& \underline{F}(z)=\sum_{z_{l}^{R}<z} r_{l},
\end{aligned}
$$

where $\bar{F}(z)$ and $\underline{F}(z)$ are upper bound and lower bound, respectively.

\section{Structural Response Analysis}

Using $p$-boxes to quantify the structural parameters, the structural response will be also a $p$-box variable. In this section, we will develop an efficient response analysis method for structures by combining $p$-box theory and the optimization method.

3.1. Sampling Random Intervals from the Dependent p-Boxes. By virtue of Sklar's theorem [18], pseudorandom samples can 
be generated from (2). That is, given a procedure to generate a sample $\left(u_{1}, u_{2}, \ldots, u_{d}\right)$ from the copula $C$, the required sample can be constructed as

$$
\begin{aligned}
& \left(x_{1}, x_{2}, \ldots, x_{d}\right) \\
& \quad=\left(F_{1}^{-1}\left(u_{1}\right), F_{2}^{-1}\left(u_{2}\right), \ldots, F_{d}^{-1}\left(u_{d}\right)\right),
\end{aligned}
$$

where $F_{i}^{-1}(i=1,2, \ldots, n)$ are quasi-inverse function of CDF and $\left(x_{1}, x_{2}, \ldots, x_{d}\right)$ are a sample from a random vector $\left(X_{1}\right.$, $\left.X_{2}, \ldots, X_{d}\right)$. So the random intervals in accordance with the dependent probability boxes can be generated by following two steps.

The first is to generate a sample from copula. There are lots of algorithms which can generate a sample from a specified copula function [19]. As conditional method may be applied for every chosen copula, it is used as the sample method and illustrated in this section. Just to explain this method in a simple way, let us assume a bivariate copula $C$ in which all of its parameters are known and pairs $(u, v)$ need to be generated from bivariate copula function $C(u, v)$. Two independent uniform $(0,1)$ variates $u$ and $t$ are generated, and then $v=$ $c_{u}{ }^{(-1)}(t)$, where $c_{u}{ }^{(-1)}(t)$ denotes a quasi-inverse of $c_{u}(v)$ which equals the partial derivative of the copula $\partial C(u, v) / \partial u$.

The other step is to compute dependent random intervals from $p$-boxes. Suppose that $\left(u_{1}, u_{2}, \ldots, u_{d}\right)$ are a sample from the copula $C$ and $\left(\left[\bar{F}_{1}\left(x_{1}\right), \underline{F}_{1}\left(x_{1}\right)\right],\left[\bar{F}_{2}\left(x_{2}\right), \underline{F}_{2}\left(x_{2}\right)\right]\right.$, $\left.\ldots,\left[\bar{F}_{d}\left(x_{d}\right), \underline{F}_{d}\left(x_{d}\right)\right]\right)$ are their CDFs. For each $u_{i}$ and corresponding to $\left[\bar{F}_{i}\left(x_{i}\right), \underline{F}_{i}\left(x_{i}\right)\right]$, a random interval is generated:

$$
x_{i}^{I}=\left[x_{i}^{L}, x_{i}^{R}\right]=\left[\bar{F}_{i}^{-1}\left(u_{i}\right), \underline{F}_{i}^{-1}\left(u_{i}\right)\right],
$$

where $x_{i}^{I}$ is random interval from $\left[\bar{F}_{i}\left(x_{i}\right), \underline{F}_{i}\left(x_{i}\right)\right]$. The superscript $I$ represents the interval distribution parameters and the superscripts $L$ and $R$, respectively, represent the lower and upper bounds of interval. $\bar{F}^{-1}$ and $\underline{F}^{-1}$ denote the quasiinverse function of upper and lower bounds of a $p$-box. Thus using $\left(u_{1}, u_{2}, \ldots, u_{d}\right)$ will simulate a range of dependent intervals $\left(x_{1}^{I}, x_{2}^{I}, \ldots, x_{d}^{I}\right)$.

Zhang et al. [9] presented an IMC method for sampling intervals from the independent $p$-boxes. When random variables contain both dependent and independent $p$-boxes, the present sampling method and the IMC method can be used to perform random sampling for dependent and independent $p$-boxes, respectively.

3.2. Metamodel-Based Interval Analysis. Through above section sampled treatments, a range of interval vectors is acquired from $p$-boxes. Consider the vector $\mathbf{x}$ of uncertain parameters defined to be contained within an interval vector (or hypercube) $\mathbf{x}^{I}$. Interval vectors sampling $m$ times from $p$ boxes are represented as $\mathbf{x}_{k}^{I}(k=1,2, \ldots, m)$. For a practical engineering problem, the input-output relationship between these parameters and the output quantity of interest is represented by the function $f(\cdot)$ applied on these parameters.
The interval analysis for the function is numerically equivalent to solving the following equation:

$$
y_{k}^{s}=\left(y_{k} \mid\left(\mathbf{x}_{k} \in \mathbf{x}_{k}^{I}\right)\left(y_{k}=f\left(\mathbf{x}_{k}^{I}\right)\right)\right),
$$

where $y_{k}^{s}$ contains all $y_{k}$ which are obtained from applying the function $f(\cdot)$ on all possible vectors within the interval vector $\mathbf{x}_{k}^{I}$. In many cases, an exact description of $y_{k}^{s}$ is extremely difficult to find. Therefore, an interval $y_{k}^{I}$ is used to approximate the exact solution $y_{k}^{s}$. For the black box model in the practical engineer problem, solution of $y_{k}^{I}$ will make the computational cost extremely expensive [20]. Besides, a great number of repeating interval analyses will be conducted, which will generate unaffordable computational burden. So metamodeling techniques are used to approximate $f(\cdot)$ for improving the computational cost.

To improve the accuracy of metamodel, design of experiments (DOE) should be applied. An experimental design domain can be acquired by truncating the $p$-box at a probability of $\alpha$ ( $\alpha$ is level of significance), which are represented as $\left[X_{i}^{L}, X_{i}^{R}\right]=\left[\bar{F}_{i}^{-1}(\alpha), \underline{F}_{i}^{-1}(1-\alpha)\right]$. The vector form is $\mathbf{X}^{I}=\left[\mathbf{X}^{L}, \mathbf{X}^{L}\right]$. In this paper, the Latin hypercube design (LHD) method is adopted, which is a space-filling design with constrainedly stratified sampling method. The sample of this method does not increase exponentially with the number of variables. Radial basis function (RBF) [21] is used to approximate the function $f(\cdot)$, which can be expressed as

$$
\widehat{f}(\mathbf{x})=\sum_{i=1}^{s} w_{i} \varphi\left(\left\|\mathbf{x}-\mathbf{x}_{i}\right\|\right),
$$

where $\mathbf{x}_{i}$ is the sampling point selected through LHS, $s$ is the number of sampling points, $w_{i}$ is mass coefficient, and $\varphi(\cdot)$ is the basis function. The common basis functions include thinplate spline, Gaussian, and multiquadrics. The metamodelbased solution of $y_{k}^{I}$ can be transferred into the following optimization problems:

$$
\begin{aligned}
& y_{k}^{L}=\min \hat{f}\left(\mathbf{x}_{k}\right), \\
& \text { s.t. } \mathbf{x}_{k}^{L} \leq \mathbf{x}_{k} \leq \mathbf{x}_{k}^{R}, \\
& y_{k}^{R}=\max \widehat{f}\left(\mathbf{x}_{k}\right), \\
& \text { s.t. } \quad \mathbf{x}_{k}^{L} \leq \mathbf{x}_{k} \leq \mathbf{x}_{k}^{R}, \\
& y_{k}^{I}=\left[y_{k}^{L}, y_{k}^{R}\right] .
\end{aligned}
$$

The gradient-based optimization techniques such as sequential quadratic programming can be used to solve this problem, and the initial point can be selected on the bound point of intervals. 
3.3. Computation of Output Quantity. Repeating $m$ times interval analysis, we will acquire intervals $y_{k}^{I}(k=1,2, \ldots$, $m)$, which can be transformed into a $p$-box by [9]

$$
P^{L} \leq P[Y \leq y] \leq P^{R}
$$

with $P^{L}=(1 / m) \sum_{k=1}^{m} \mathbf{I}\left[y_{k}^{R} \leq y\right]$ and $P^{R}=(1 / m) \sum_{i=1}^{m} \mathbf{I}\left[y_{k}^{L} \leq\right.$ $y$ ], where $I$ is the indicator function. Summarizing the above procedure, the present method can be summarized as follows.

Step 1. Collect all $p$-boxes $\mathbf{X}=\left(X_{1}, X_{2}, \ldots, X_{n}\right)$ and sample $m$ interval vectors $\mathbf{x}_{i}^{I}=\left(x_{i, 1}^{I}, x_{i, 2}^{I}, \ldots, x_{i, n}^{I}\right)(i=1,2, \ldots, m)$ from $\mathbf{X}$ by the method in Section 3.1.

Step 2. Conduct the design of experiments with LHS in the experimental design domain $\left[\mathbf{X}^{L}, \mathbf{X}^{R}\right]$.

Step 3. Construct a RBF response surface for approximating (7).

Step 4. Conduct optimization analysis over each $\mathbf{x}_{i}^{I}$ and acquire interval vector $\mathbf{y}^{I}$.

Step 5. Generate the $p$-box of the output quantity by (10).

\section{Error Analysis}

The absolute error and the relative error are popular to measure the accuracy between the experimental data and the true value, which is extended to analysis errors of different $p$ boxes. In this paper, the absolute errors contain the area error and the boundary error. Suppose that the two $p$-boxes $A=\left[\bar{F}_{A}(x), \underline{F}_{A}(x)\right]$ and $B=\left[\bar{F}_{B}(x), \underline{F}_{B}(x)\right]$; then the left area error and the right area error between two $p$-boxes are, respectively, calculated by

$$
\begin{aligned}
& S^{L}=\int_{-\infty}^{\infty}\left|\bar{F}_{A}(x)-\bar{F}_{B}(x)\right| d x, \\
& S^{R}=\int_{-\infty}^{\infty}\left|\underline{F}_{A}(x)-\underline{F}_{B}(x)\right| d x .
\end{aligned}
$$

The area error between two $p$-boxes is defined as the sum of the left area error and the right area error, which is written as $S=S^{L}+S^{R}$. As the area error cannot reflect the discrepancy of boundary between two $p$-boxes, the discrepancy of the boundary is used as the boundary error. Plotting a line with the cumulative probability $\alpha$ will produce two intersection points with the boundary of a $p$-box. For $\left[\bar{F}_{A}(x), \underline{F}_{A}(x)\right]$, two intersection points are $\bar{F}_{A}^{-1}(\alpha)$ and $\underline{F}_{A}^{-1}(\alpha)$. Two intersection points of $\left[\bar{F}_{B}(x), \underline{F}_{B}(x)\right]$ are $\bar{F}_{B}{ }^{-1}(\alpha)$ and $\underline{F}_{B}{ }^{-1}(\alpha)$. Thus the boundary error $\Delta L(\alpha)$ is

$$
\Delta L(\alpha)=\Delta L_{1}(\alpha)+\Delta L_{2}(\alpha)
$$

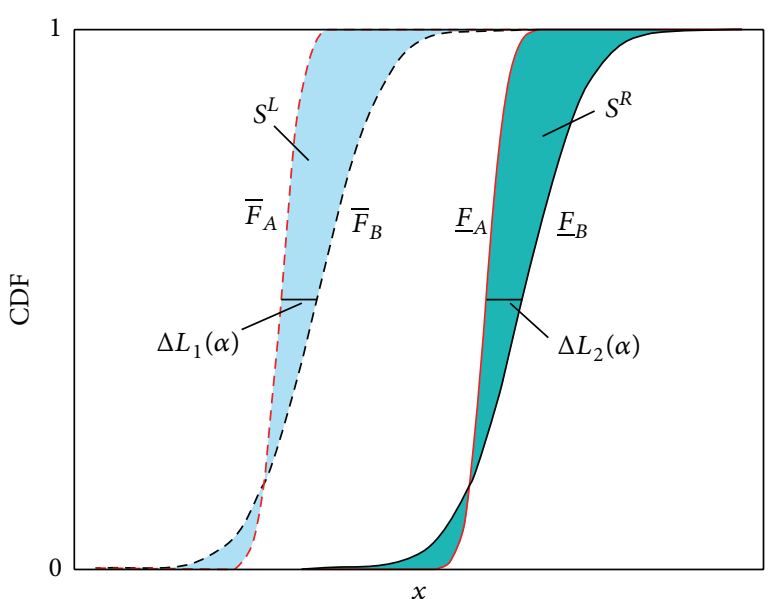

FIGURE 1: Schematic diagram of the area error and the boundary error between $\left[\bar{F}_{A}, \underline{F}_{A}\right]$ and $\left[\bar{F}_{B}, \underline{F}_{B}\right]$.

where $\Delta L_{1}(\alpha)=\left|\bar{F}_{A}^{-1}(\alpha)-\bar{F}_{B}^{-1}(\alpha)\right|$ and $\Delta L_{2}(\alpha)=$ $\left|\underline{F}_{A}{ }^{-1}(\alpha)-\underline{F}_{B}{ }^{-1}(\alpha)\right|$. The illustration of the area error and the boundary area is shown in Figure 1.

The relative error is used for comparing the precision of different $p$-boxes, which have the relative area error and the relative boundary error. Just to explain the definition, two $p$-boxes are named, respectively, the relative $p$-box and the contrastive $p$-box. The definition of relative area error is the absolute error of two $p$-boxes dividing the area between two bounds of the relative $p$-box. Suppose that $\left[\bar{F}_{A}, \underline{F}_{A}\right]$ and $\left[\bar{F}_{B}, \underline{F}_{B}\right]$ are taken as the relative $p$-box and the contrastive $p$-box, respectively. The relative area error is defined by

$$
E_{R}=100 * \frac{|S|}{\int_{-\infty}^{\infty}\left|\bar{F}_{A}(x)-\underline{F}_{A}(x)\right| d x} \%,
$$

where $S$ is the area error between two $p$-boxes. The relative boundary error in the probability value $\alpha$ is computed by

$$
E_{L}(\alpha)=100 * \frac{|\Delta L(\alpha)|}{\left|\bar{F}_{A}^{-1}(\alpha)-\underline{F}_{A}^{-1}(\alpha)\right|} \%,
$$

where $\Delta L(\alpha)$ is the boundary error between two $p$-boxes. Two types of error are used to quantify the errors between $p$-boxes, when the discrepancy of different $p$-boxes can be distinguished from the figure.

\section{Numerical Examples and Discussions}

5.1. Sum of $X$ and $Y$. The test function

$$
Z=X+Y \text {, }
$$

where $X$ and $Y$ are random variables represented by dependent $p$-boxes with the Gaussian copula. The Pearson correlation $\rho$ of the two random variables is $0.3 . X$ is bounded by normal distribution whose means are between 2 and 8 
TABLE 1: Uncertain parameters for the 10-bar aluminum truss.

\begin{tabular}{lccc}
\hline Uncertain variables & Parameter 1 (mean) & Parameter 2 (standard deviation) & Boundary parameter distribution \\
\hline$F_{1}(\mathrm{~N})$ & {$[442800,44680]$} & 40000 & Normal \\
$F_{2}(\mathrm{~N})$ & {$[442800,44680]$} & 40000 & Normal \\
$F_{3}(\mathrm{~N})$ & {$[1709200,1849200]$} & 40000 & Normal \\
$E(\mathrm{Mpa})$ & 68948 & {$[3120,3350]$} & Normal \\
\hline
\end{tabular}

and whose standard deviation is 0.5 . $Y$ also is specified by the class of normal distributions with means $[15,20]$ and standard deviation 1.5. According to the statistics theory, the theoretical cumulative probability of $Z$ can be calculated by the following equations:

$$
=\int_{-\infty}^{z} \frac{1}{\sqrt{2 \pi\left(\sigma_{1}^{2}+2 \rho \sigma_{1} \sigma_{2}+\sigma_{2}^{2}\right)}} e^{\left(z-\mu_{1}^{I}-\mu_{2}^{I}\right)^{2} / 2\left(\sigma_{1}^{2}+2 \rho \sigma_{1} \sigma_{2}+\sigma_{2}^{2}\right)} d z,
$$

where $\mu_{1}^{I}=[2,8], \mu_{2}^{I}=[15,20], \sigma_{1}=0.5$, and $\sigma_{2}=1.5$. The numerical boundaries of $Z$ are computed by the Simpson quadrature [22].

As a comparison, the DBC method with a range of the discretization numbers $\mathbf{M}=[10,20, \ldots, 400]$ is adopted to compute this problem here. According to the Cartesian product, $\mathbf{M}^{2}$ analyses would be required if two $p$-boxes are discretized into $\mathbf{M}$ focal elements, respectively. The absolute error values with the theoretical $p$-boxes under the different discretization number are shown in Figures 2 and 3 by the red line with asterisk markers. Now, use the present method with the sample sizes $\mathbf{M}^{2}$ to solve the same problem, and their absolute error values are plotted in Figures 2 and 3 with the blue line with filled circular markers.

From the area error lines in Figure 2, two lines have the obvious convergence procedure with the increasing iterations, while the area error of the present method has the faster convergence procedure. Under the same calculated amount, the circular-marked values are always under the asteriskmarked values. In other words, the present method has more accuracy than the DBC method. Figure 3 shows the boundary error line under three boundary points $\alpha=0.025,0.5$, and 0.975 with two different methods. These boundary error lines have the trend of approximate 0 with increasing the calculation time. In three boundary points, the filled circularmarked error lines are always below the asterisk-marked error lines, except little points when $\alpha=0.5$ that the asteriskmarked values are slightly bigger than the circular-marked lines. Using the present method will produce less error than using the DBC method under the same computation time; thus the present method has more calculation efficiency. For the multiple random variables, this advantage is more obvious. The calculated amount using the DBC method is exponential growth with increasing number of random variables and discretization number. This numerical test demonstrated the validity of the proposed present method. The calculation of structural responses is performed in the following section using the present method.

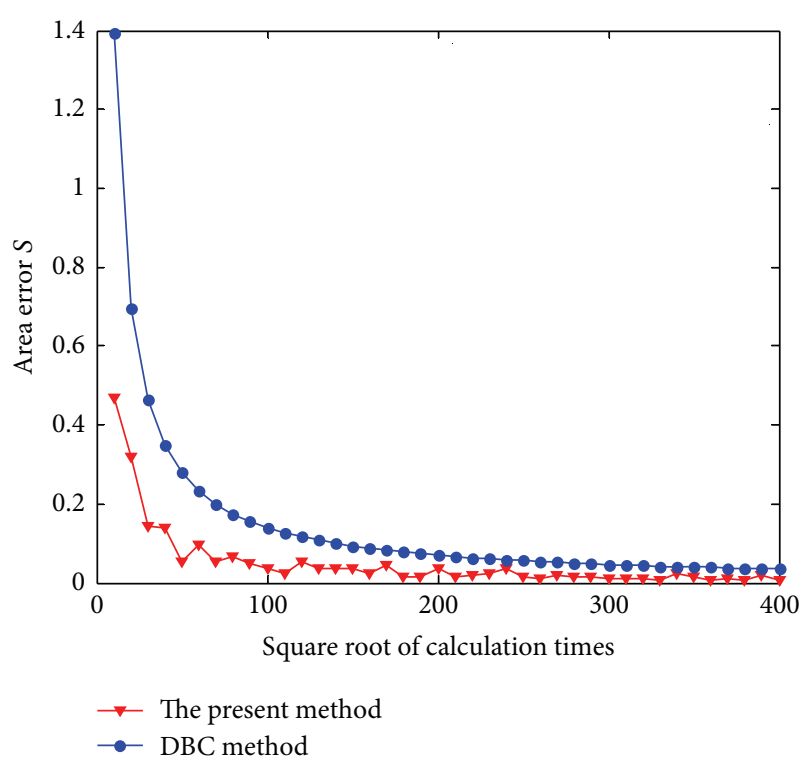

FIgURE 2: Area error lines with the present method and the DBC method.

5.2. A 10-Bar Aluminum Truss. A linear elastic 10-bar aluminum truss [23] is shown in Figure 4. The certain parameters for the 10-bar aluminum truss are the density $\rho=$ $2768 \mathrm{~kg} / \mathrm{m}^{3}$, the length of the horizontal and vertical bars $L=9.144 \mathrm{~m}$, and the bars areas $A=4000 \mathrm{~mm}^{2}(i=1,2$, $\ldots, 10)$. The truss is subjected to two vertical forces $F_{1}$ and $F_{2}$, and a horizontal force $F_{3}$, which are uncertain variables of the structures. The Young modulus $E$ is treated as the random variables. The interesting system response is $d_{y}$ the vertical displacement of the joint 2 . The uncertain parameters presented by $p$-boxes are shown in Table 1.

$F_{1}, F_{2}, F_{3}$, and $E$ are truncated with $\alpha=0.05$ as the design domain of input data. LHS method is applied to generate sampling points and finite element method is used to solve $d_{y}$. RBF approximation model is constructed, which can be written as

$$
d_{y}=f\left(F_{1}, F_{2}, F_{3}, E\right)
$$

where $F_{2}$ and $F_{3}$ are dependent and the Young modulus $E$ and the force $F_{1}$ are independent variables. Dependence in $F_{2}$ and $F_{3}$ is represented by the Gaussian copula with Pearson coefficient 0.9 . The probability bounds of $d_{y}$ are computed 


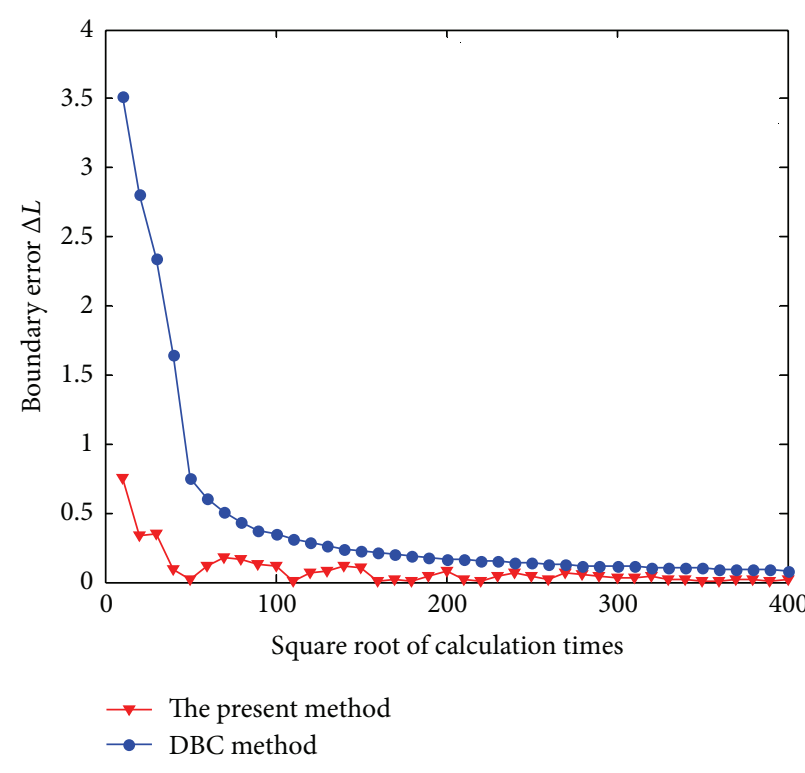

(a) Boundary error lines using two methods with $\alpha=0.025$

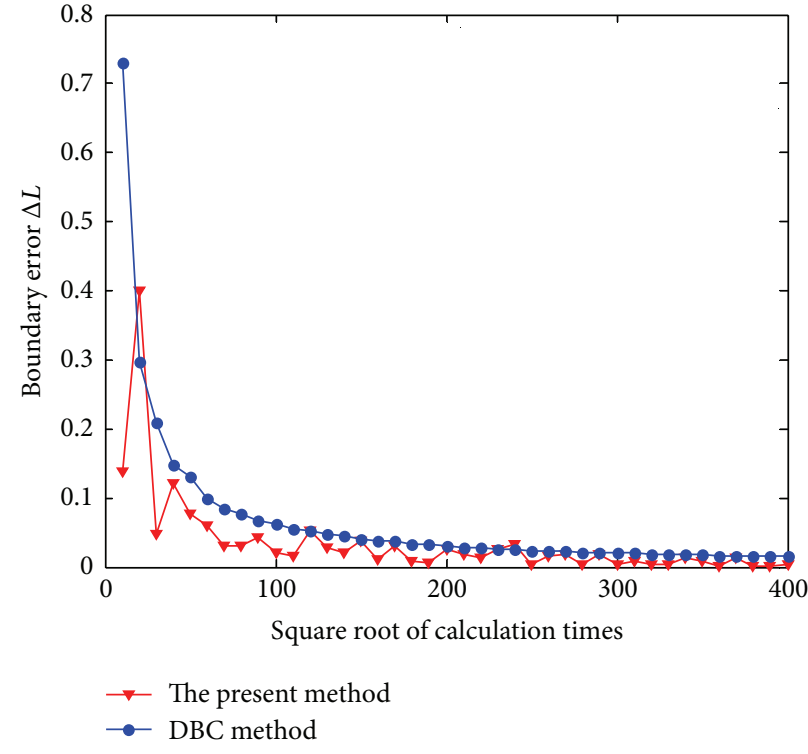

(b) Boundary error lines using two methods with $\alpha=0.5$

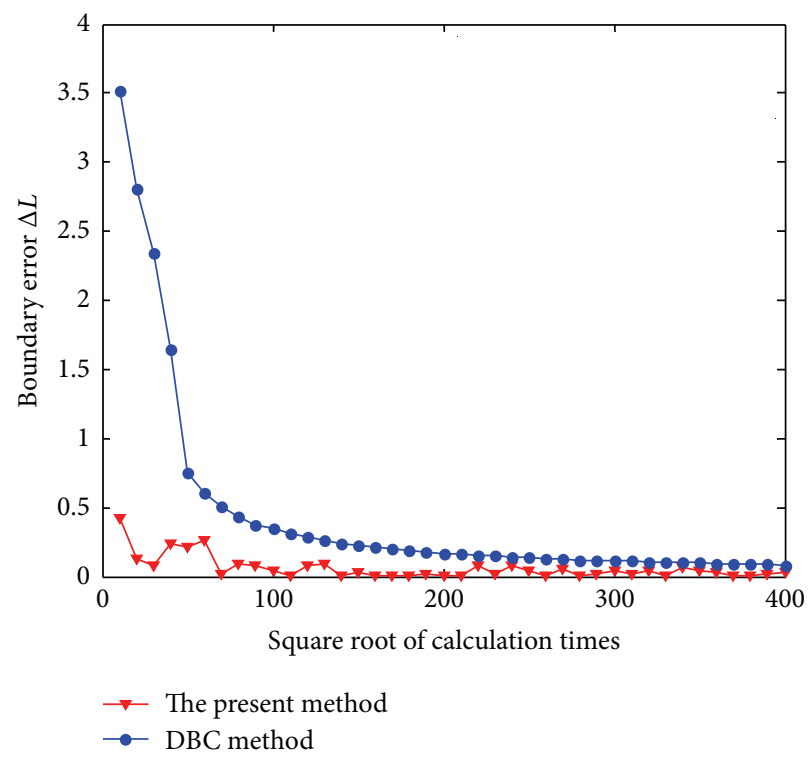

(c) Boundary error lines using two methods with $\alpha=0.975$

FIgURE 3: Boundary error lines with $\alpha=0.025,0.5$, and 0.975 .

by the present method involving by $10^{5}$ replications. For comparison purpose, we assume that $F_{1}, F_{2}, F_{3}$, and $E$ are mutually independent due to lacking enough dependent information. The result of $d_{y}$ is also calculated by the present method with $10^{5}$ sample size. Two $p$-boxes of $d_{y}$ considering dependent or independent input data are plotted in Figure 5 which are called $\left[\bar{F}_{A}, \underline{F}_{A}\right]$ and $\left[\bar{F}_{B}, \underline{F}_{B}\right]$, respectively. $\left[\bar{F}_{A}, \underline{F}_{A}\right]$ is regarded as the theoretical value and $\left[\bar{F}_{B}, \underline{F}_{B}\right]$ is taken as proximate value due to lacking enough dependent information. The relative errors between $\left[\bar{F}_{A}, \underline{F}_{A}\right]$ and $\left[\bar{F}_{B}, \underline{F}_{B}\right]$ are shown in Table 2. It can be found that the relative area error is $3.43 \%$. When $\alpha=0.025$ or $\alpha=0.975$, the boundary error are $8.24 \%$ and $7.85 \%$, respectively. It can be noticed that the relative boundary error is close to zero when $\alpha=0.5$. From this example, the dependence in the input $p$-boxes has great impact on probability bounds of structural responses, which is particularly obvious in the tail of probability bounds.

5.3. A 25-Bar Steel Truss. This case is a 25-bar steel truss [24] which is shown in Figure 6. The length of each horizontal or vertical bar is $15.24 \mathrm{~m}$ and the cross-sectional area of all bars is $5000 \mathrm{~mm}^{2}$. The Poisson ratio is 0.3 . The joint 12 is hingesupported, and the joints 6,8 , and 10 are roller-supported. The joints 7,9 , and 11 are subjected to the vertical loads $F_{3}, F_{2}$, 
TABLE 2: Relative area error and relative boundary errors between the theoretical $p$-box and the approximate $p$-box.

\begin{tabular}{lc}
\hline Error type & $\begin{array}{c}\text { The theoretical } p \text {-box and the } \\
\text { approximate } p \text {-box }\end{array}$ \\
\hline $\begin{array}{l}\text { Relative area error } \\
\text { Relative boundary error }\end{array}$ & $3.43 \%$ \\
$\alpha=0.025$ & $8.24 \%$ \\
$\begin{array}{l}\text { Relative boundary error } \alpha=0.5 \\
\text { Relative boundary error } \\
\alpha=0.975\end{array}$ & $0.01 \%$ \\
\hline
\end{tabular}

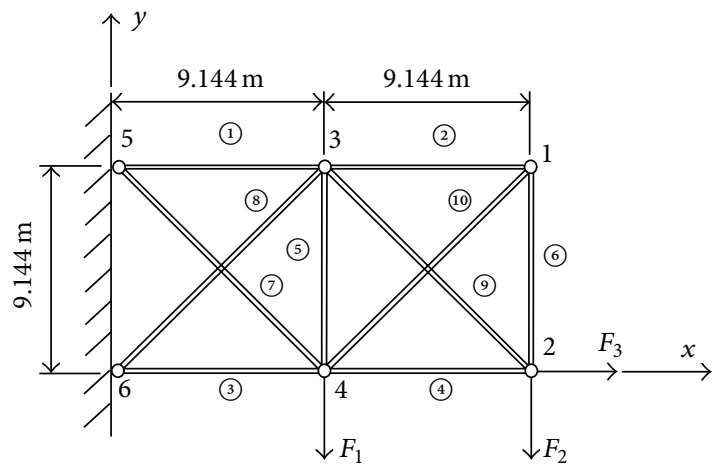

Figure 4: A 10-bar aluminum truss.

and $F_{1}$, respectively, and the joint 1 is subjected to a horizontal load $F_{4}$. The forces $F_{1}, F_{2}, F_{3}$, and $F_{4}$ and the Young modulus $E$ are treated as $p$-boxes, which are shown in Table 3 . The interesting value is the vertical displacement $d_{3}$ of the joint 9. RBF is used to replace the finite element model, which can be written as

$$
d_{3}=f\left(F_{1}, F_{2}, F_{3}, F_{4}, E\right) \text {, }
$$

where $F_{1}, F_{2}, F_{3}$, and $F_{4}$ and $E$ are truncated at the probability of 0.05 and LHS is used to sample points.

Four cases are designed to analyse the influence from dependence in input variables and shown in Table 4. For cases 1-3, each of two forces is mutually dependent and their copulas are Frank copula. $E$ is regarded as the independent variable. The relatively large correlation coefficient 0.9 for random variables is used. In case 4 , five input variables are assumed to be mutually independent. $p$-boxes of $d_{3}$ in four cases are obtained by the present method with $10^{4}$ samples that are shown in Figure 7 , where $p$-boxes of $d_{3}$ with the condition in cases 1-3 are taken as the theoretical value and $p$-box of $d_{3}$ with case 4 is used as approximate value. The relative area error between the theoretical value and approximate value is listed in Table 5. By comparing the relative error under different cases, the relative area error or the relative bounds area under case 2 is more than the error under case 1 and case 3 . For each case 1 -case 3 , the relative bounds error for $\alpha=0.5$ is less than the relative bounds error for $\alpha=0.05$ or $\alpha=0.95$, which is very small and

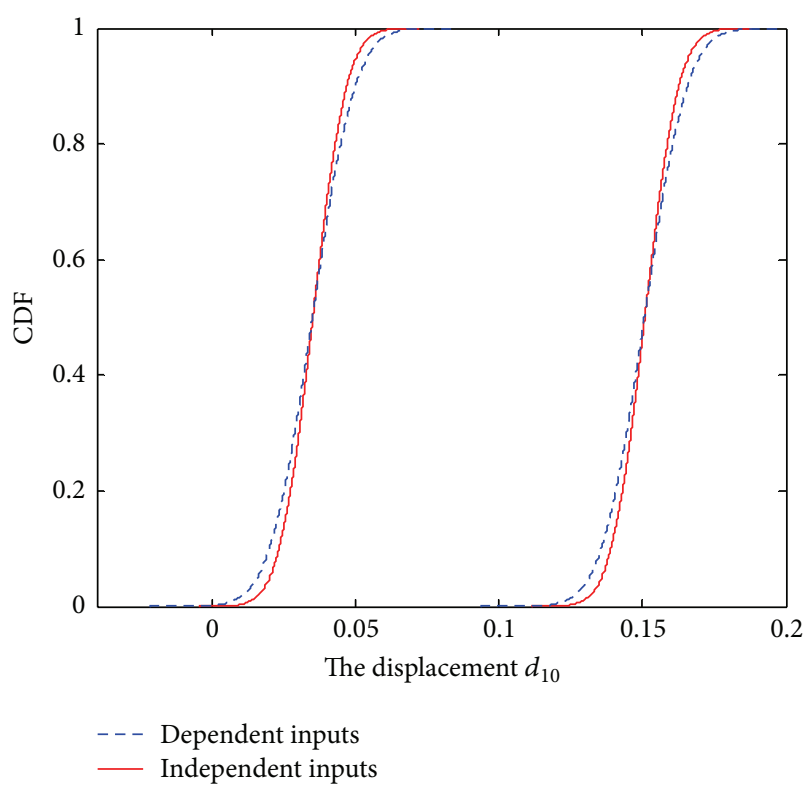

FIGURE 5: $p$-boxes of $d_{10}$ with dependent and independent inputs.

nearly 0 . Overall, dependence under case 2 has more influence for the structural response of $d_{3}$ in this example. This example demonstrates that neglecting the dependence in input variables will produce unpredictable error for bounds of structural response.

5.4. Application to a Taylor Impact Test. Taylor impact tests are often performed to confirm the plastic behavior of a material under severe strain conditions. Extremely high plastic strains and strain rates occur at the crushed end of the rod, resulting in severe local deformation. The experimental data usually consist of measurements of its final deformed profile. As shown in Figure 8, the cylindrical sample total length $L_{\text {initial }}=3.8 \mathrm{~cm}$ and the diameter $D_{\text {initial }}=0.76 \mathrm{~cm}$. Testing material is the high-strength low-alloy (HSLA-100) steel [25]. The modulus of elasticity is taken to be $E=$ $197 \mathrm{GPa}$ and the material density $\rho=7842 \mathrm{~kg} / \mathrm{m}^{3}$. The initial velocity of the sample is $246 \mathrm{~m} / \mathrm{s}$. For the Taylor test, the final deformed profile is an important index to evaluate the material performance. Thus in the application the uncertainty response analysis is conducted for the final total length $L_{\text {final }}$ and the final diameter $D_{\text {final }}$. To compute the two values, the finite element method is employed, and Zerilli-Armstrong (Z-A) model

$$
\sigma=\alpha_{1}+\alpha_{2} \exp \left[\left(-\alpha_{3}+\alpha_{4} \ln \frac{\partial \varepsilon}{\partial t}\right) T\right]+\alpha_{5} \varepsilon^{\alpha_{6}}
$$

is used for rate-dependent plasticity, where $\varepsilon$ is the equivalent plastic strain, $\sigma$ is resulting stress, and $T$ is the temperature at the start of the experiment. The parameters $\alpha_{1} \sim \alpha_{6}$ are material-specific. The Belytschko-Tsay shell element is used to create finite element model with 2000 elements. The initial temperature $T$ is $298 \mathrm{~K}$. The time duration of the impact process is $20 \mathrm{~ms}$. 


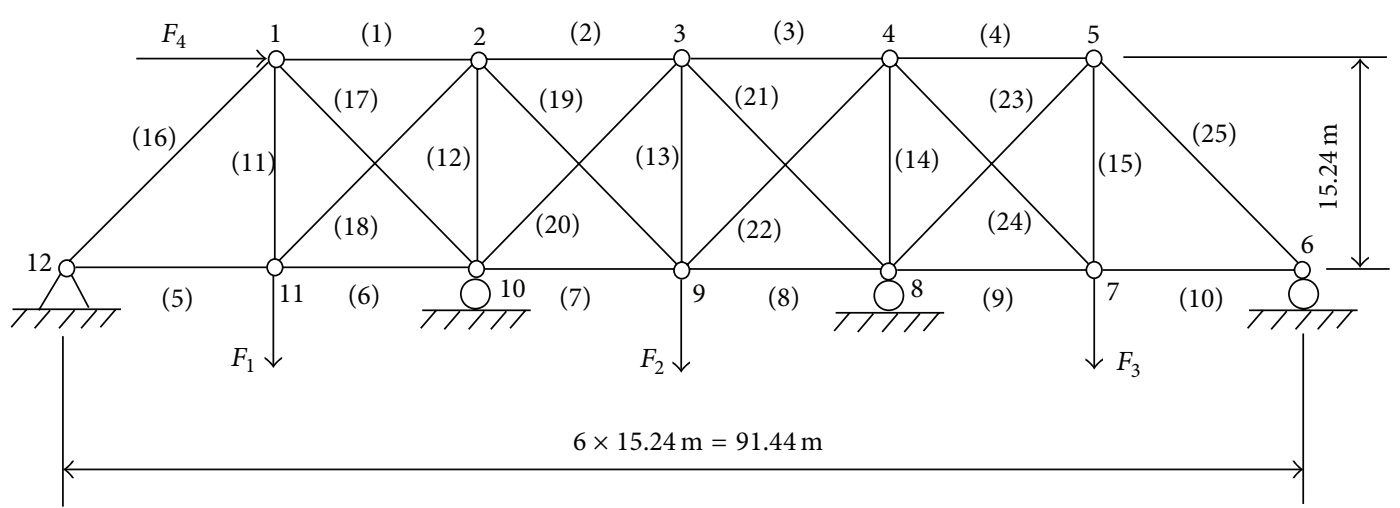

FIGURE 6: A 25-bar steel truss [24].

TABLE 3: Uncertain variables for the 25-bar steel truss.

\begin{tabular}{lccc}
\hline Uncertain variables & Parameter $1($ mean) & Parameter 2 (standard deviation) & Boundary distribution type \\
\hline$F_{1}(\mathrm{~N})$ & {$[1.679 e 6,1.879 e 6]$} & $3.2 e 5$ & Normal \\
$F_{2}(\mathrm{~N})$ & {$[2.124 e 6,2.324 e 6]$} & $3.2 e 5$ & Normal \\
$F_{3}(\mathrm{~N})$ & {$[1.679 e 6,1.879 e 6]$} & $3.2 e 5$ & Normal \\
$F_{4}(\mathrm{~N})$ & {$[1.234 e 6,1.434 e 6]$} & $3.2 e 5$ & Normal \\
$E(\mathrm{Mpa})$ & {$[1.99949 e 5,2.10125 e 5]$} & $1.949 e 3$ & Normal \\
\hline
\end{tabular}

TABLE 4: Four cases under different dependence.

\begin{tabular}{ll}
\hline Dependent case number & Dependency and Kendall correlations \\
\hline Case 1 & Kendall coefficient between $F_{1}$ and $F_{2}$ is $\tau_{1,1}=0.9$ \\
\hline Case 2 & Kendall coefficient between $F_{3}$ and $F_{4}$ is $\tau_{1,2}=0.9$ \\
\hline Case 3 & Kendall coefficient between $F_{1}$ and $F_{3}$ is $\tau_{2,1}=0.9$ \\
& Kendall coefficient between $F_{2}$ and $F_{4}$ is $\tau_{2,2}=0.9$ \\
\hline Case 4 & Kendall coefficient between $F_{1}$ and $F_{4}$ is $\tau_{3,1}=0.9$ \\
\hline
\end{tabular}

TABLE 5: Relative area error and relative boundary errors under four cases.

\begin{tabular}{lccc}
\hline Error type & Case 1 and case 4 & Case 2 and case 4 & Case 3 and case 4 \\
\hline Relative area error & $3.80 \%$ & $13.62 \%$ & $3.31 \%$ \\
Relative boundary error $\alpha=0.025$ & $11.58 \%$ & $35.99 \%$ & $8.39 \%$ \\
Relative boundary error $\alpha=0.5$ & $0.46 \%$ & $0.24 \%$ & $1.19 \%$ \\
Relative boundary error $\alpha=0.975$ & $7.77 \%$ & $29.81 \%$ & $12.97 \%$ \\
\hline
\end{tabular}

TABLE 6: Distribution of the random variables for the Taylor test.

\begin{tabular}{lccc}
\hline Z-A parameters & Distribution parameter 1 & Distribution parameter 2 & Distribution type \\
\hline$\alpha_{1}(\mathrm{MPa})$ & $\mu_{\alpha_{1}}=[86.5,119.5]$ & $\sigma_{\alpha_{1}}=4.125$ & Normal \\
$\alpha_{2}(\mathrm{MPa})$ & $\mu_{\alpha_{2}}=[922.5,985.5]$ & $\sigma_{\alpha_{2}}=7.875$ & Normal \\
$\alpha_{3}\left(\mathrm{~K}^{-1}\right)$ & $\mu_{\alpha_{3}}=[3.8 e-4,4.4 e-4]$ & $\sigma_{\alpha_{3}}=7.375 e-5$ & Normal \\
$\alpha_{4}\left(\mathrm{~K}^{-1}\right)$ & $\mu_{\alpha_{4}}=[1.025 e-4,1.315 e-4]$ & $\sigma_{\alpha_{4}}=3.625 e-6$ & Normal \\
$\alpha_{5}(\mathrm{MPa})$ & $\mu_{\alpha_{5}}=[985,1007]$ & $\sigma_{\alpha_{5}}=2.75$ & Normal \\
$\alpha_{6}$ & $\mu_{\alpha_{6}}=[0.2365,0.575]$ & $\sigma_{\alpha_{6}}=0.0026$ & Normal \\
\hline
\end{tabular}


TABLE 7: Correlation coefficient matrix for the Z-A model parameters.

\begin{tabular}{lcccccc}
\hline Z-A parameters & $\alpha_{1}$ & $\alpha_{2}$ & $\alpha_{3}$ & $\alpha_{4}$ & $\alpha_{5}$ \\
\hline$\alpha_{1}$ & 1 & -0.083 & 0.372 & 0.207 & -0.488 & 0.267 \\
$\alpha_{2}$ & -0.083 & 1 & 0.344 & 0.311 & 0.082 \\
$\alpha_{3}$ & 0.372 & 0.344 & 1 & & 0.130 \\
$\alpha_{4}$ & 0.207 & 0.311 & 0.802 & 1 & -0.653 \\
$\alpha_{5}$ & -0.488 & 0.082 & 0.453 & 0.271 & -0.271 & 1 \\
$\alpha_{6}$ & 0.267 & 0.130 & -0.621 & -0.466 & -0.860 \\
\hline
\end{tabular}

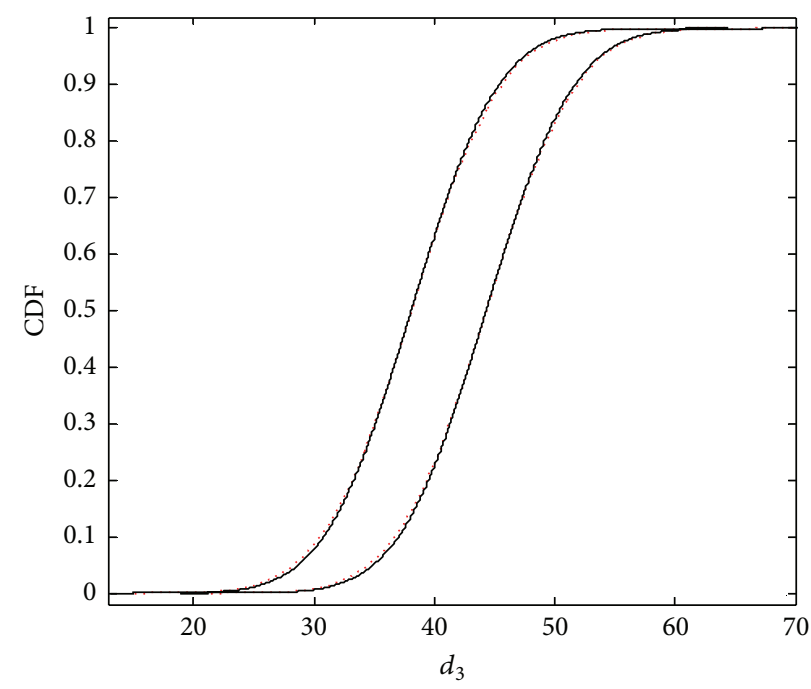

p-box in case 4

- $p$-box in case 1

(a) $p$-boxes for $d_{3}$ with case 1 and case 4

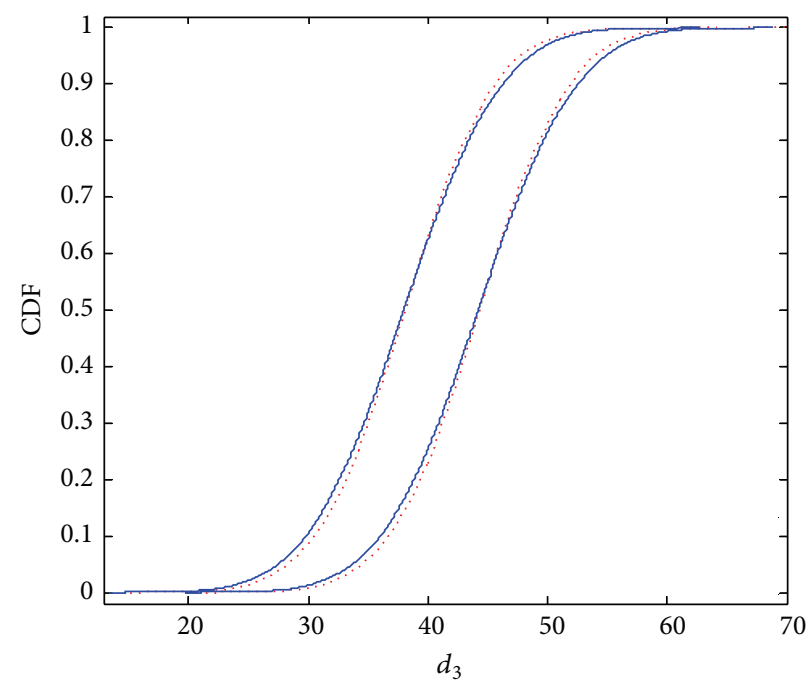

..... p-box in case 4

- $p$-box in case 2

(b) $p$-boxes for $d_{3}$ with case 2 and case 4

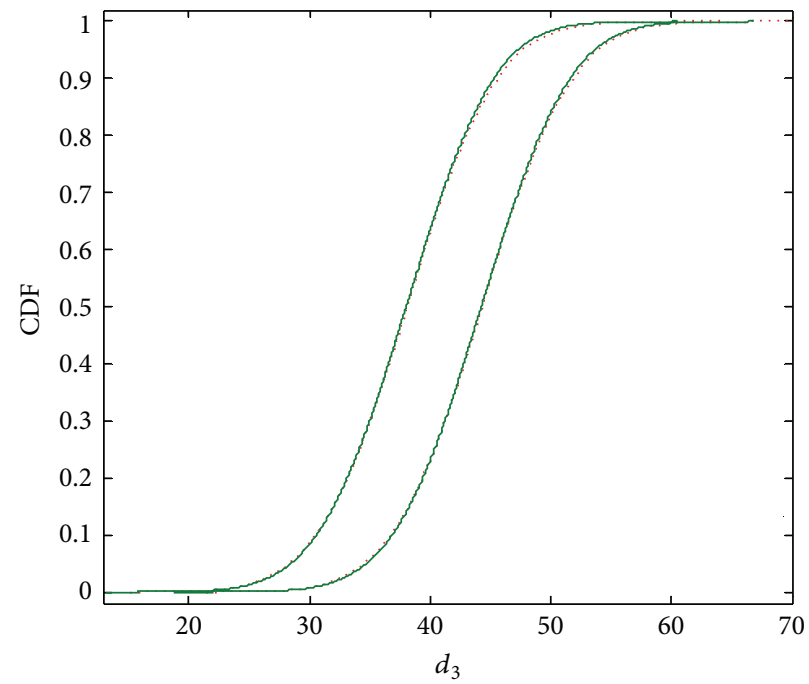

p-box in case 4

_ $p$-box in case 3

(c) p-boxes for $d_{3}$ with case 3 and case 4

Figure 7: $p$-boxes for $d_{3}$ in three cases. 


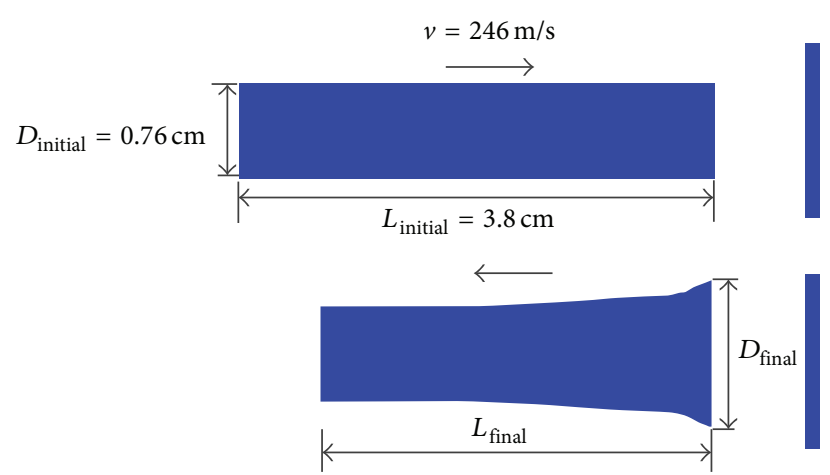

FIgURE 8: Simulating the Taylor impact test.

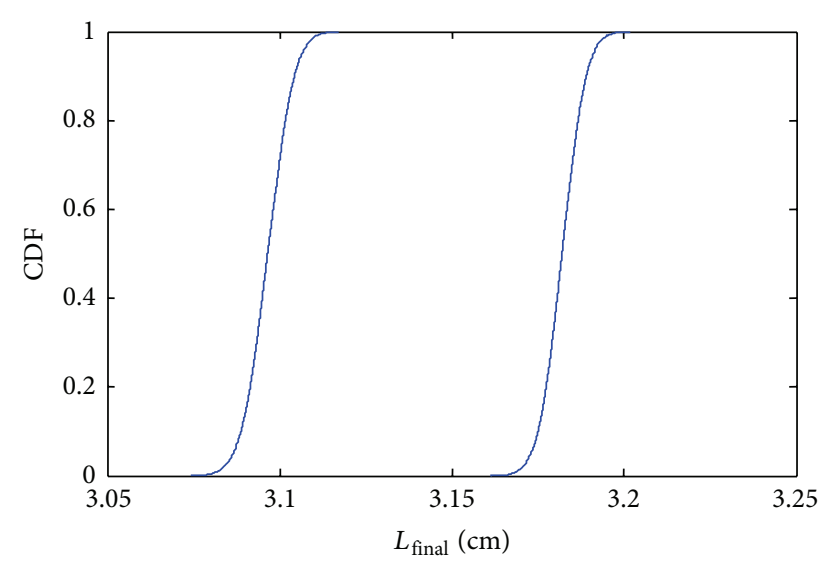

Figure 9: $p$-boxes of $L_{\text {final }}$.

Due to the manufacturing and measuring errors, $\mathrm{Z}-\mathrm{A}$ model parameters are thus treated as $p$-boxes variables, which are given in Table 6 . There is dependence in these parameters [26] and their correlation coefficients are given in Table 7.

According to the present method, the random variables are truncated at $\alpha=5 e-4$ firstly. For generating the sample points, LHS method is used. The approximation models of $L_{\text {final }}$ and $D_{\text {final }}$ are constructed by RBF, which are expressed as

$$
\begin{aligned}
& L_{\text {final }}=f\left(\alpha_{1}, \alpha_{2}, \alpha_{3}, \alpha_{4}, \alpha_{5}, \alpha_{6}\right), \\
& D_{\text {final }}=g\left(\alpha_{1}, \alpha_{2}, \alpha_{3}, \alpha_{4}, \alpha_{5}, \alpha_{6}\right) .
\end{aligned}
$$

The analysis results of dependence are plotted in Figures 9 and 10, respectively. From this example, the present method is also effective for the nonlinear model including dependent uncertain parameters.

\section{Conclusion}

A computational method has been developed for the calculation of the structural responses under uncertain parameters represented by dependent $p$-boxes. The $p$-boxes are discretized into a range of intervals based on the copula sampling technique. The calculation of structural response interval is

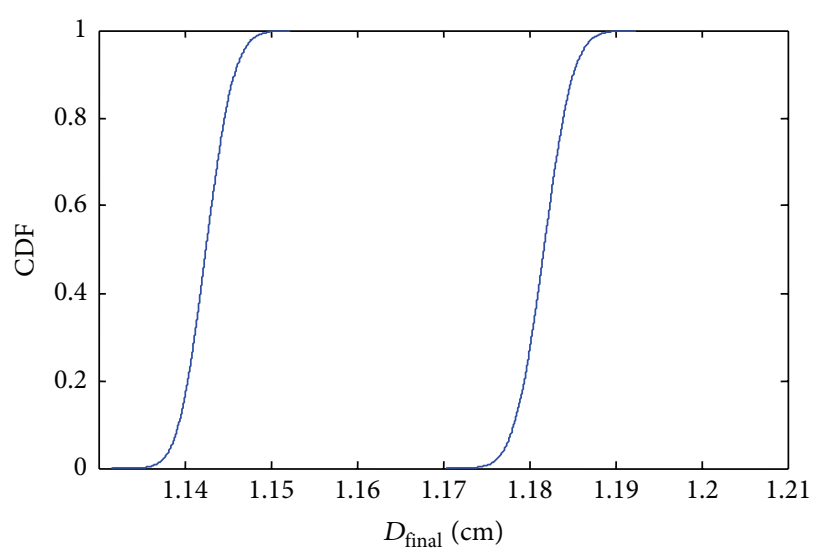

FIGURE 10: $p$-boxes of $D_{\text {final }}$.

carried out by the metamodel-based optimization method. The resulting intervals are then assembled into a $p$-box. The present method takes both the advantages of efficiency of sampling method and the advantage of handling dependent p-boxes of DBC method.

By investigating dependence in the structural uncertainty parameters based on the present method, dependence in the variables cannot be neglected. When the dependent variables are regarded as the independent variables, the probability bound of structural responses will produce error, which are unpredictable and may lead to the failure decision-making. We have demonstrated, through the use of the present method for solving a benchmark problem and three numerical examples, that this method is effective and practical for the structural response analysis.

\section{Conflict of Interests}

The authors declare that there is no conflict of interests regarding the publication of this paper.

\section{Acknowledgment}

This work is supported by the grant from the Fundamental Research Funds for the Central Universities (Grant no. 227201401203).

\section{References}

[1] R. E. Moore, Methods and Applications of Interval Analysis, SAIM Publisher, Philadelphia, Pa, USA, 1979.

[2] R. R. Yager, J. Kacprzyk, and M. Fedrizzi, Advances in the Dempster-Shafer Theory of Evidence, John Wiley \& Sons, New York, NY, USA, 1994.

[3] K. Sentz and S. Ferson, "Combination of evidence in DempsterShafer theory," SAND 2002-0835, Sandia National Laboratories, Albuquerque, NM, USA, 2002.

[4] M. Beer, S. Ferson, and V. Kreinovich, "Imprecise probabilities in engineering analyses," Mechanical Systems and Signal Processing, vol. 37, no. 1-2, pp. 4-29, 2013. 
[5] J. M. Aughenbaugh and C. J. J. Paredis, "The value of using imprecise probabilities in engineering design," Transactions of the ASME, Journal of Mechanical Design, vol. 128, no. 4, pp. 969979, 2006.

[6] R. C. Williamson and T. Downs, "Probabilistic arithmetic. I. Numerical methods for calculating convolutions and dependency bounds," International Journal of Approximate Reasoning, vol. 4, no. 2, pp. 89-158, 1990.

[7] D. R. Karanki, H. S. Kushwaha, A. K. Verma, and S. Ajit, "Uncertainty analysis based on probability bounds (P-Box) approach in probabilistic safety assessment," Risk Analysis, vol. 29, no. 5, pp. 662-675, 2009.

[8] M. C. Bruns, Propagation of imprecise probabilities through black box models [M.S. thesis], Georgia Institute of Technology, Atlanta, Ga, USA, 2006.

[9] H. Zhang, R. L. Mullen, and R. L. Muhanna, "Interval Monte Carlo methods for structural reliability," Structural Safety, vol. 32, no. 3, pp. 183-190, 2010.

[10] H. Zhang, R. L. Mullen, and R. L. Muhanna, "Finite element structural analysis using imprecise probabilities based on p-box representation," in Proceedings of the 4th International Workshop on Reliable Engineering Computing 'Robust Design-Coping with Hazards, Risk and Uncertainty', pp. 3-5, National University of Singapore, Singapore, March 2010.

[11] H. Zhang, R. L. Mullen, and R. L. Muhanna, "Structural analysis with probability-boxes," International Journal of Reliability and Safety, vol. 6, no. 1-3, pp. 110-129, 2012.

[12] Y. C. Bai, C. Jiang, X. Han, and D. A. Hu, "Evidence-theorybased structural static and dynamic response analysis under epistemic uncertainties," Finite Elements in Analysis and Design, vol. 68 , pp. 52-62, 2013.

[13] D. D. Ghosh and A. Olewnik, "Computationally efficient imprecise uncertainty propagation," Journal of Mechanical Design, vol. 135, no. 5, Article ID 051002, 12 pages, 2013.

[14] W. E. Vesely, F. F. Goldberg, N. H. Roberts, and D. F. Hassl, Fault Tree Handbook, U.S. Nuclear Regulatory Commission, Washington, DC, USA, 1987.

[15] J. W. Hickman, "PRA procedures guide: a guide to the performance of probabilistic risk assessments for nuclear power plants, in two volumes," NUREGCR-2300-V1 and-V2, National Technical Information Service, Washington, DC, USA, 1983.

[16] K. Hanson and F. Hemez, "Uncertainty quantification of simulation codes based on experimental data," in Proceedings of the 41st Aerospace Sciences Meeting and Exhibit, American Institute of Aeronautics and Astronautics, 2003.

[17] S. Ferson, R. B. Nelsen, J. Hajagos et al., "Dependence in probabilistic modeling, Dempster-Shafer theory, and probability bounds analysis," SAND 2004-3072, Sandia National Laboratories, 2004.

[18] R. B. Nelsen, An Introduction to Copulas, vol. 139 of Lecture Notes in Statistics, Springer, Berlin, Germany, 1999.

[19] U. Cherubini, E. Luciano, and W. Vecchiato, Copula Methods in Finance, John Wiley \& Sons, New York, NY, USA, 2004.

[20] D. Moens and M. Hanss, "Non-probabilistic finite element analysis for parametric uncertainty treatment in applied mechanics: recent advances," Finite Elements in Analysis and Design, vol. 47, no. 1, pp. 4-16, 2011.

[21] M. D. Buhmann, Radial Basis Functions: Theory and Implementations, vol. 12 of Cambridge Monographs on Applied and Computational Mathematics, Cambridge University Press, Cambridge, UK, 2003.
[22] W. Gander and W. Gautschi, "Adaptive quadrature-revisited," BIT Numerical Mathematics, vol. 40, no. 1, pp. 84-101, 2000.

[23] P. Yi, G. Cheng, and L. Jiang, "A sequential approximate programming strategy for performance-measure-based probabilistic structural design optimization," Structural Safety, vol. 30, no. 2, pp. 91-109, 2008.

[24] C. Jiang, X. Han, and G. R. Liu, "Optimization of structures with uncertain constraints based on convex model and satisfaction degree of interval," Computer Methods in Applied Mechanics and Engineering, vol. 196, no. 49-52, pp. 4791-4800, 2007.

[25] R. L. Martineau, M. B. Prime, and T. Duffey, "Penetration of HSLA-100 steel with tungsten carbide spheres at striking velocities between 0.8 and $2.5 \mathrm{~km} / \mathrm{s}$," International Journal of Impact Engineering, vol. 30, no. 5, pp. 505-520, 2004.

[26] K. M. Hanson and F. M. Hemez, "Uncertainty quantification of simulation codes based on experimental data," in Proceedings of the 41st Aerospace Sciences Meeting and Exhibit, January 2003. 


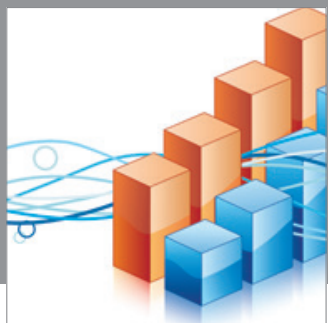

Advances in

Operations Research

mansans

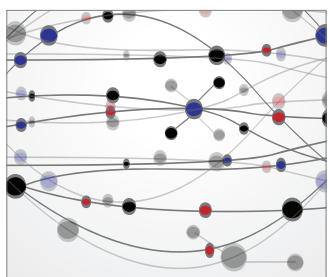

The Scientific World Journal
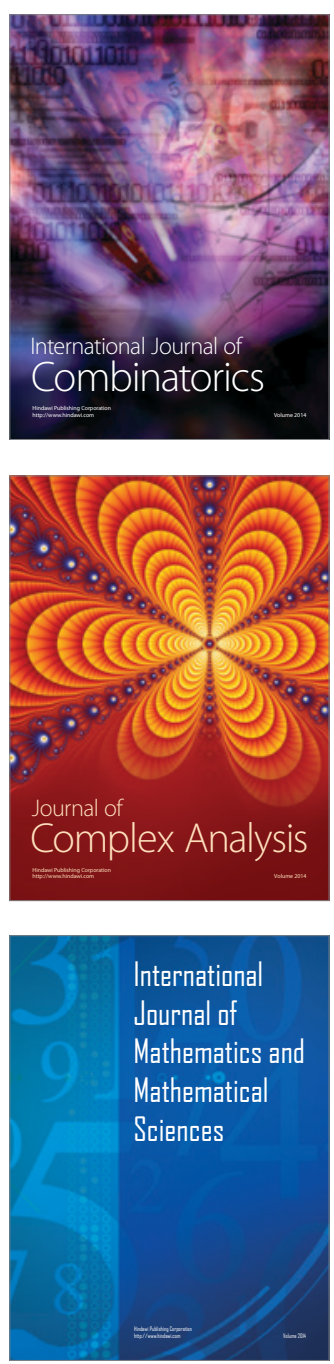
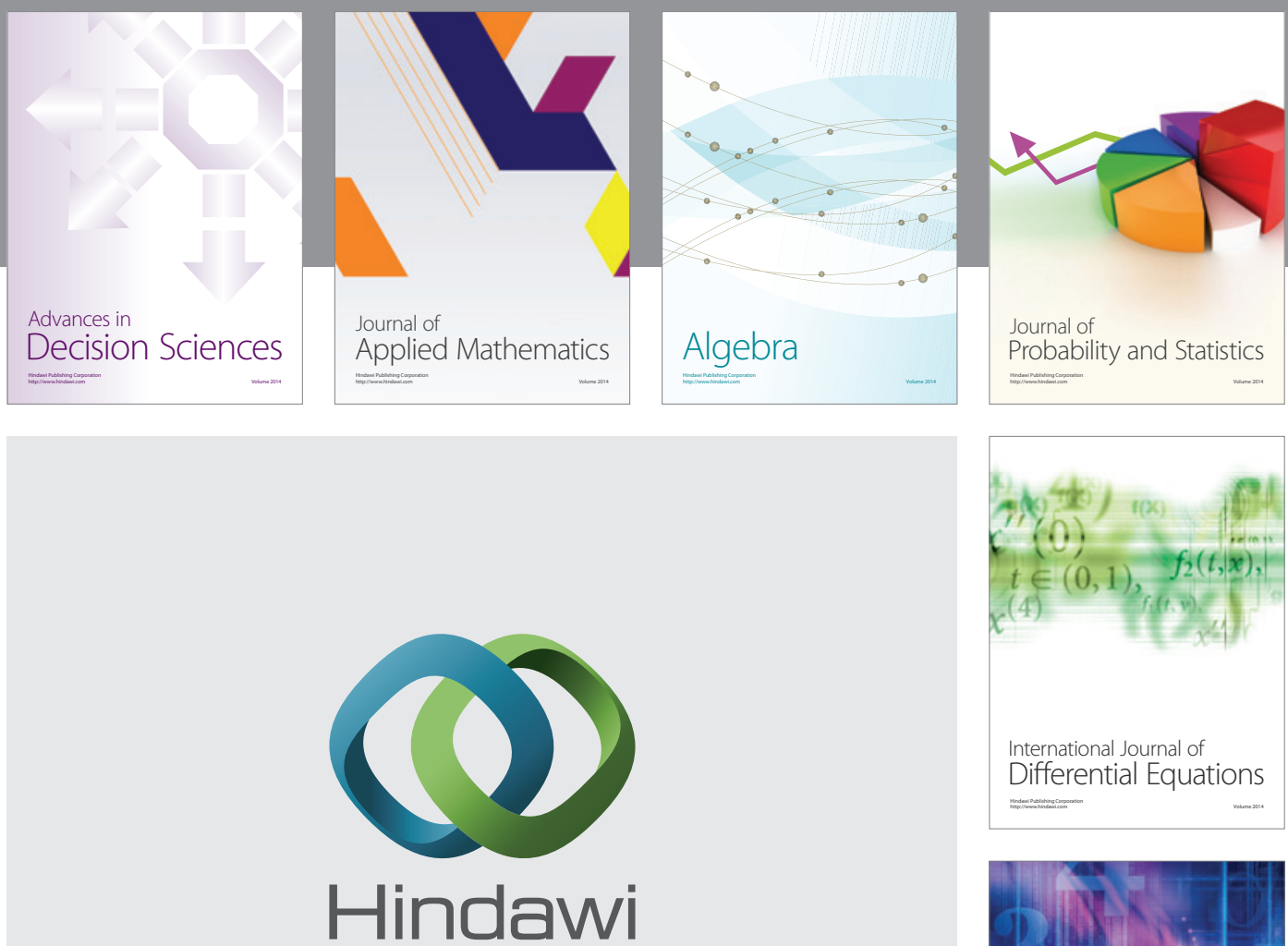

Submit your manuscripts at http://www.hindawi.com
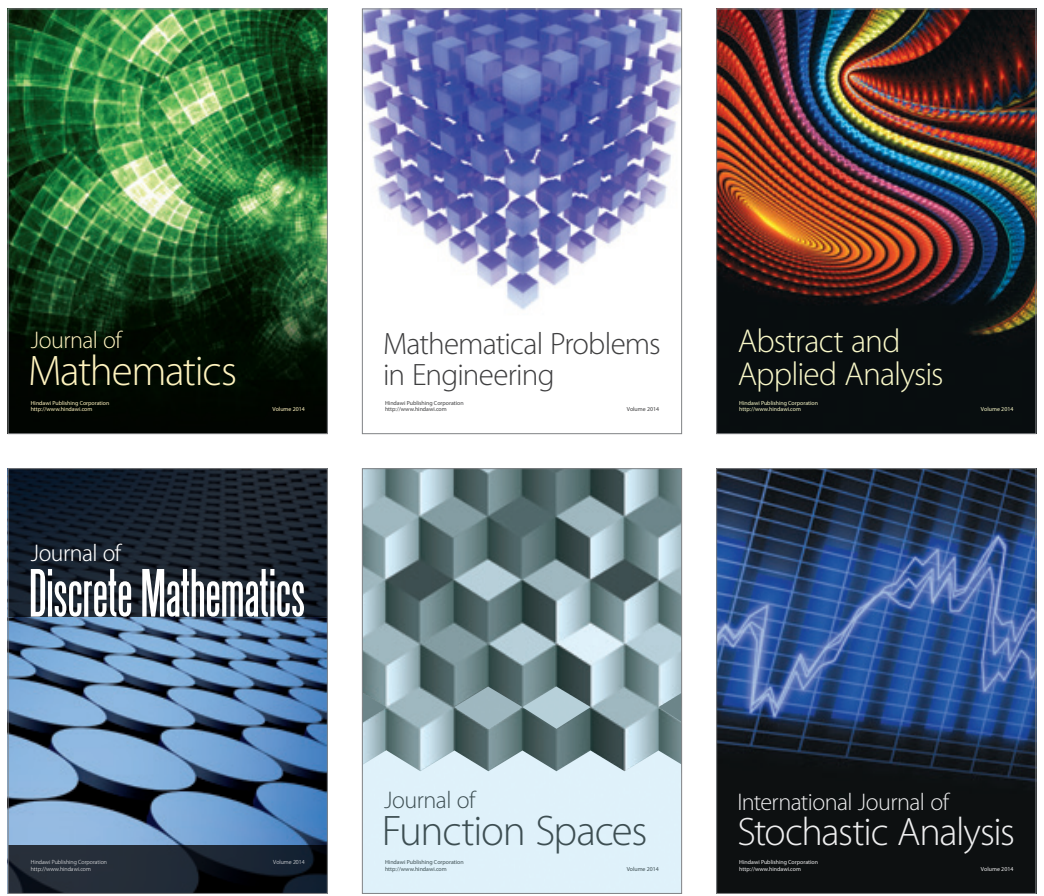

Journal of

Function Spaces

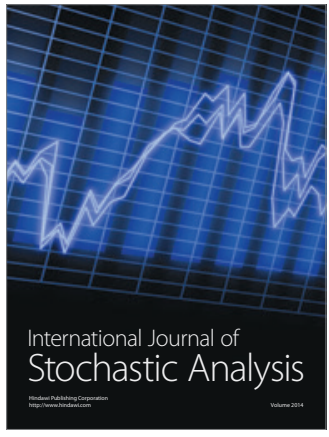

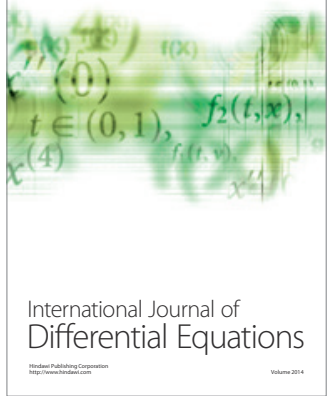
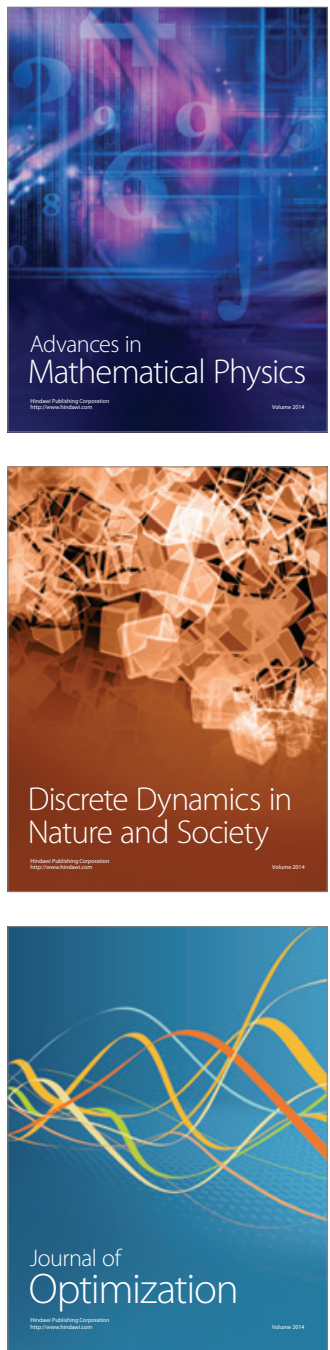